\title{
Gestión de la atención médica, herramienta fundamental para los médicos residentes
}

\author{
Management of medical care, a fundamental tool for resident doctors \\ Guillermo Fajardo-Ortiz ${ }^{*}$ y Héctor Robledo \\ ${ }^{1}$ Facultad de Medicina, Universidad Nacional Autónoma de México; 2 Instituto de Oftalmología Conde de la Valenciana. Ciudad de México, México
}

\section{Resumen}

En el área de la enseñanza médica, y particularmente dentro de nuestro país, las residencias médicas en hospitales son el mejor modelo para realizar un posgrado; sin embargo, cuando un médico joven inicia su residencia y arriba a un hospital, difícilmente posee los conocimientos de los servicios, tipo de pacientes y procesos que se realizan en dicho lugar, lo que repercute directamente en el cumplimiento de sus tareas, aprendizaje y, más importante, los servicios que ofrece el hospital. Por lo tanto, es imperante hacer partícipes a los médicos residentes en la gestión de la atención médica, darles a conocer que esta es la encargada de articular de forma armónica todos los procesos médico-administrativos en los que participan pacientes, recursos humanos, materiales y financieros, para alcanzar el cumplimiento de la misión y la visión hospitalaria con eficiencia operativa y sentido humano. De esta manera será posible orientarlos a realizar una correcta toma de decisiones $y$ una gestión adecuada de los recursos, y a que su contribución en los procesos médico-administrativos brinde un servicio de calidad y seguridad para el paciente.

PALABRAS CLAVE: Gerencia. Médico residente. Procesos. Residencias médicas. Competencias.

\begin{abstract}
In the area of medical education, and particularly in our country, medical residencies are the best educational programs for a graduated physicians; however, when young medical doctors begin a residence at hospitals, they hardly poses knowledge about the way medical services work as well as the processes they involve, which directly affects the fulfillment of their tasks, their process of learning and more importantly, it interferes in the services provided by the hospital. Therefore, it is imperative to immerse residents in the management of medical care and let them know that its main function is to harmoniously articulate every medical-administrative process related to patients as well as human, material and financial resources. One of the main goals is to achieve the fulfillment of the hospital's mission and vision with operational efficiency and humanism. This path will help physicians to make the best decisions, as well as achieving an adequate management of resources always remembering that quality in medical services and patient's safety are important.
\end{abstract}

KEY WORDS: Management. Resident physicians. Process. Medical residencies. Competitions.

\author{
Correspondencia: \\ *Guillermo Fajardo-Ortiz \\ Fac. Medicina, UNAM \\ Ciudad Universitaria \\ C.P. 04510, Ciudad de México, México \\ E-mail: gfortiz@unam.mx
}

Fecha de recepción: 06-12-2017

Fecha de aceptación: 27-01-2018

DOI://dx.doi.org/10.24875/CIRU.M18000005
Cir Cir. 2018;86:71-78

Disponible en PubMed www.cirugiaycirujanos.com 


\section{Introducción}

El tema se inicia con una interrogante: ¿por qué es necesario que los médicos residentes tengan conocimiento sobre gestión de la atención médica? Es indudable que personas del medio clínico y otros profesionales de la salud desconocen sus ventajas o la consideran innecesaria, pero entender el funcionamiento de dicho instrumento ayuda a generar un servicio eficaz, eficiente y de calidad. Los médicos residentes son un pilar fundamental en la atención médica, pues están en contacto estrecho con los pacientes y su institución, y conocen sus problemas. En el desempeño de sus actividades clínicas y de docencia, implícitamente practican actividades de gestión, en particular en cuanto a procesos médico-administrativos, debiendo tomar decisiones correctas y oportunas. Al promover una cultura de gestión en los médicos residentes se pretende aprovechar en forma óptima los recursos, obteniendo así mejores resultados en la atención médica, en especial en los hospitales.

\section{Gestión hospitalaria}

El hospital es el establecimiento de atención médica tradicional y óptimo para realizar la residencia médica; sin embargo, para muchos estudiosos de la administración es la organización más compleja por diversas razones. Una de ellas es la diversidad de pacientes que atiende, personas por las que existe la atención médica, y dicha diversidad responde a diferentes situaciones: problemas de salud-enfermedad, edad, sexo, nivel socioeconómico y cultura. La segunda razón es que cuenta con capital humano muy heterogéneo en relación con la formación académica y la preparación técnica, la experiencia, la edad, las jornadas de trabajo, el sexo, los sueldos o salarios, y las conductas e ideas. Y una tercera razón hace referencia a los recursos materiales y tecnológicos de la atención médica, encontrándose variedad en equipo, mobiliario e instrumental, tamaño, costo, durabilidad, mantenimiento y ubicación, de tal manera que hay desde piezas pequeñas, como pueden ser un lápiz, un bisturí o una computadora, hasta grandes como el inmueble, y los hay costosos como un equipo de tomografía axial computarizada y baratos como una hoja de papel. En otros términos, en el hospital hay grandes disparidades; es un conjunto de componentes humanos, sociales, científicos, tecnológicos, económicos y políticos de aspectos culturales y tradición diversos, intervinculados de manera transdisciplinaria y sistémica, con valores compartidos, lo que significa gestión, la cual debe articular de forma armónica todos los procesos médico-administrativos en que participan pacientes, recursos humanos, materiales y financieros, orientándolos al logro de la misión y la visión hospitalarias con eficiencia operativa y sentido humano. La misión del hospital se refiere a lograr objetivos de la institución con eficacia, ética y calidad; es decir, la misión es el motivo de la existencia del establecimiento. Por otra parte, la visión es la forma en que el hospital se visualiza en un futuro determinado, es una prospectiva. Dichos atributos son las bases para garantizar la gobernanza y la excelencia de la gestión, lo que implica profesionalización, desburocratización, control de gastos, productividad, competitividad, rentabilidad, indicadores de costo-beneficio, planificación estratégica, aplicación de competencias, valoración de resultados e innovaciones tecnológicas; todas estas actividades y otras más orientadas al bienestar del paciente.

\section{Niveles de gestión hospitalaria}

La gestión hospitalaria comprende tres niveles según el nivel jerárquico en que se práctica:

- Macrogestión: se refiere a la política institucional, a las decisiones de autoridades superiores, directores, subdirectores o administradores, que están delimitadas por la normatividad vigente; coordina actividades generales, se orienta a la utilización óptima de todos los recursos, incluyendo el tiempo y la planeación a largo plazo; tiene un conocimiento global de los procesos de los departamentos y servicios; afecta a los otros dos niveles, y se proyecta extrainstitucionalmente. Este nivel de gestión tiene vinculación y alineación, cuando es el caso, con políticas y lineamientos sectoriales o gubernamentales.

- Mesogestión: se identifica con las actividades de las autoridades de mandos medios, jefes de unidad, coordinadores de servicios y departamentos tales como recepción, admisión, calidad y seguridad del paciente, consulta externa, trabajo social, servicio al cliente (al usuario), relaciones públicas, terapia intensiva, estudios auxiliares de diagnóstico y tratamiento, recursos (talento) humanos, enfermería, mantenimiento, ingeniería biomédica, contabilidad y finanzas, tecnología de la información y las comunicaciones, 
contraloría interna, etc. Determina procesos intrainstitucionales.

- Microgestión o gestión clínica: se refiere a las acciones realizadas por el personal médico (incluyendo a los residentes), paramédico y técnico, con relación básicamente a los pacientes, o sea, la realización de procesos médico-administrativos. La microgestión es de gran importancia, pues es en ella donde se puede poner en riesgo la vida del paciente, ya sea por falta de control o de implementación de las mejores prácticas en cada uno de los servicios, por no seguir los procesos de manera adecuada o por falta de supervisión de los responsables jerárquicos en la estructura.

La articulación entre los niveles jerárquicos puede ser vertical, horizontal, zigzagueante y virtual. La vertical se orienta de los puestos directivos superiores a las bases, o viceversa; la horizontal se refiere a la vinculación de actividades del mismo nivel; la zigzagueante relaciona diversos niveles de orden jerárquico; y la virtual es digital y se manifiesta con rapidez en todos los niveles.

\section{Los procesos médico-administrativos en la gestión clínica}

Los procesos médico-administrativos en la gestión clínica se refieren a la conjunción de recursos para satisfacer las necesidades y demandas institucionales y de los pacientes de forma complementaria y lógica. De acuerdo con la teoría general de sistemas, transforman los componentes de entrada (insumos) en resultados (exsumos), aportando valores añadidos o extra para los pacientes y el organismo. Los insumos se refieren a todos los recursos: personal (formación académica, preparación técnica, actitud, ideas, experiencia, talento, capacidad de resolución de problemas, trato, interés, motivación, vocación de servicio, disposición de trabajo en equipo, etc.), normativa vigente, recursos y compromisos financieros, equipo tecnológico de diagnóstico y tratamiento médico, instalaciones, métodos, tiempo, etc. Los exsumos son los productos: egresos de pacientes, costos finales, recursos utilizados, tiempo empleado, etc. El propósito de los procesos es ofrecer un servicio adecuado, que cubra las necesidades y satisfaga las expectativas de todos los involucrados en aspectos de calidad, seguridad, eficacia y eficiencia; para que se logre, es necesario que quienes practiquen la gestión posean cuatro cualidades paralelas:
- Responsabilidad por su propio trabajo.

- Compromiso con los pacientes, el personal, la institución y la comunidad.

- Iniciativa y capacidad de toma de decisiones.

- Aceptación de retos y, en su caso, presentación de propuestas de cambio.

Para llevar a cabo los procesos en la microgestión es necesario que los médicos residentes tengan conocimientos en cuanto a:

- Precisión y definición de los procesos en los diferentes servicios y situaciones: admisión, consulta externa, referencia-contrarreferencia, elaboración de documentos, etc., teniendo en cuenta que los procesos son multifactoriales. Es muy importante que el residente participe en los procesos de manera informada, acatando la normativa en la materia, descrita en los lineamientos del Consejo de Salubridad General, para actuar en consistencia con la calidad y la seguridad del paciente.

- Coordinación de recursos de los procesos participantes; por ejemplo, realización de estudios antes de los procedimientos quirúrgicos.

- Organización del personal, conformación de equipos de trabajo y determinación de locales, equipo, instrumental y material para la realización de los procesos.

- Tiempos de realización de los procesos.

\section{El médico residente}

Hay que recordar: "La residencia es reconocida como la mejor modalidad para la formación médica de posgrado»'. El hospital es el lugar preferente para llevar a cabo la residencia, ámbito de adiestramiento en la adquisición de diversas habilidades clínicas y quirúrgicas. En México, de acuerdo con la Ley Federal de Trabajo, se entiende por «Médico residente: el profesional de la medicina con título legalmente expedido y registrado ante las autoridades competentes a una unidad médica receptora de residentes, para cumplir con una residencia». En la citada ley se expresa que residencia es: «El conjunto de actividades que deba cumplir un médico residente en periodo de adiestramiento; para realizar estudios y prácticas de posgrado, respecto de la disciplina de la salud a que pretenda dedicarse, dentro de una unidad médica receptora de residentes, durante el tiempo y conforme a los requisitos que señalan las disposiciones académicas vigentes»². De acuerdo con lo anterior, la residencia médica es un periodo 
de formación en el que el médico residente está en etapa de aprendizaje, realizando actividades clínicas y quirúrgicas, en las que tácitamente hay actos de gestión; la mayoría de las veces, por no decir siempre, los médicos residentes carecen de conocimientos respecto a la misma.

Cuando los residentes -médicos jóvenes- llegan a un hospital o a un área clínica para iniciar sus actividades, arriban a un ambiente y una cultura en gran parte desconocidos, y van con deseos de aprender, superarse y prestar servicios. En ocasiones se les orienta en cuanto a sus labores, se les hace una recepción, un acto de bienvenida con explicaciones sobre la institución, se les encauza en aspectos médico-administrativos; esto sucede en el mejor de los escenarios, pero en otras ocasiones se les asigna de inmediato una actividad clínica-docente, sin que los médicos residentes conozcan los servicios, los procesos ni el tipo de pacientes. En cualquier caso, perciben autoridades y jerarquías, necesidad de cumplir con normas y procesos médico-administrativos, por lo que deben aprender los valores, las reglas y los diversos procedimientos propios de la institución.

De acuerdo con lo señalado en la microgestión, particularmente en los procesos médico-administrativos, los médicos residentes deben conocer su institución en cuanto a la organización, el personal, las jerarquías, los recursos físicos, los locales, el inmueble, las actividades clínicas que se realizan y las actividades docentes y de investigación. Esto favorece su adaptación a los servicios, así como el cumplimiento de los procesos, que deben estar estructurados con el fin de lograr unas mayores eficacia y eficiencia de los recursos humanos, técnicos, económicos y administrativos. El desarrollo de los procesos de forma adecuada propicia los menores inconvenientes y costos para el usuario, las instituciones, los seguros médicos y la sociedad. Acorde con lo expresado, en particular en cuanto a costos, se ha señalado que "los profesionales sanitarios (fundamentalmente médicos clínicos) asignan el $70 \%$ de los recursos sanitarios en sus decisiones diagnósticas y terapéuticas, realizadas en condiciones de incertidumbre; lograr una adecuada gerencia clínica es una tarea que supone coordinar recursos y motivar personas. La gestión clínica pretende restaurar los niveles de salud de los pacientes, en la medida de lo posible, mediante acciones que conciernen a pacientes individuales 0 a grupos de pacientes (agrupados por una afección o un procedimiento comunes, por la unidad de asistencia, etc.). Las decisiones directas sobre cada paciente las adopta el médico o la enfermera, trabajando por separado o en equipo, y las decisiones que afectan a grupos de pacientes son tomadas principalmente por los responsables asistenciales. La disciplina fundamental de la gestión clínica es la medicina» ${ }^{3}$.

Los procesos en que participan los médicos residentes involucran acciones clínicas, docentes y de tipo administrativo que se practican en todos los servicios y áreas dentro del hospital: consulta externa, hospitalización, urgencias, quirófano, laboratorio de análisis clínicos, imagenología, diálisis peritoneal, cirugía robótica, etc.

En el ámbito hospitalario, en términos reduccionistas puede expresarse que los procesos son un conjunto de actividades desarrolladas entre profesionales y pacientes ${ }^{4}$. Dichos procesos deben favorecer las competencias formativas, fomentar la creatividad y la innovación por parte de los médicos residentes, y su conocimiento es necesario especialmente en situaciones problemáticas:

- Falta de asistencia, supervisión o asesoría de médicos de jerarquía mayor, debiendo actuar con iniciativa propia de acuerdo con la lex artis ad hoc; el médico residente es responsable de sus propios actos. Este aspecto es de suma importancia, particularmente ante el crecimiento acelerado de la medicina defensiva, en la que cada vez más los abogados buscan dónde estuvo el error, la falla o el descuido para justificar la argumentación de su cliente en pos de un arreglo económico.

- Tomar decisiones propias no adecuadas a su nivel formativo, no acatando las indicaciones 0 instrucciones ordenadas por médicos o autoridades superiores.

El residente médico requiere estar atento a las situaciones problemáticas, con una mente analítica y propositiva, teniendo presente que en numerosas ocasiones debe servir y laborar en áreas que no pertenecen a su ámbito habitual, cumplir jornadas prolongadas o realizar actividades no planeadas, imprevistas, ante la dinámica cambiante del hospital. Son momentos de duda, de reflexión, de incertidumbre, de planear y tomar decisiones; son situaciones delicadas no solo para los médicos residentes, sino también para los pacientes y los organismos de atención médica. En estos casos, los médicos residentes deben demostrar su capacidad de gestión y aplicación o creación de los procesos para alinear su experiencia, prácticas y 
habilidades con las expectativas de otras áreas. En el camino van descubriendo nuevos senderos, reinventando procesos.

Un tema que no puede pasarse por alto es el agotamiento y el cansancio de los residentes, ya que afectan la gestión y el funcionamiento de los procesos, pero sobre todo incrementan el riesgo en materia de seguridad de los pacientes en los servicios médicos. Los médicos residentes suelen presentar el síndrome de desgaste profesional, determinado por el estrés laboral al que se encuentran sometidos y que se caracteriza por cansancio emocional, actos violentos, despersonalización, baja autoestima, insatisfacción o conflictos laborales, desmotivación y desinterés por las actividades. Dicho síndrome puede afectar la calidad de los servicios médicos y la enseñanza ${ }^{5}$. Esta situación puede mejorarse si los médicos residentes poseen y aplican la microgestión y los procesos.

\section{PUEM y Norma: educación en salud para la organización y el funcionamiento de las residencias médicas}

Los médicos residentes, en su desempeño laboral y docente en formación, están normados en México por el Plan Único de Especializaciones Médicas (PUEM) y por la Norma Oficial Mexicana NOM-001SSA3-2012 titulada Educación en salud para la organización y funcionamiento de residencias médicas. El PUEM es el ordenamiento de las obligaciones y del desarrollo de los alumnos (residentes) en los cursos de especialización. Es un documento elaborado en la Facultad de Medicina de la Universidad Nacional Autónoma de México, señalando que la finalidad del mismo es formar especialistas competentes en los diversos campos del saber y el quehacer de la medicina, capaces de llevar a cabo una práctica profesional de alta calidad, que integran a su trabajo cotidiano de atención médica las actividades de investigación y educación. EI PUEM contiene disposiciones generales, obligaciones de los alumnos, derechos de los alumnos, faltas en las que incurren los alumnos, sanciones, reconocimientos y estímulos, y se expresan definiciones, disposiciones y actividades de los residentes ${ }^{6}$.

En la Norma Oficial, en el apartado 4, hay definiciones que son importantes para la microgestión y los procesos médico-administrativos:

4.1 Guardia, conjunto de actividades descritas y calendarizadas en el programa operativo, adicionales a las que el médico residente debe realizar durante la jornada laboral, en la unidad médica receptora de residentes en la que está adscrito o asignado.

4.2 Institución de salud, toda organización con personalidad jurídica de derecho público o privado, con capacidad para ofertar servicios de salud y responder de su orden, estructura y prestaciones en uno o más establecimientos para la atención médica.

4.3 Jornada laboral, número de horas que, en días hábiles, el médico residente debe desarrollar las funciones y actividades señaladas en el programa operativo de la residencia médica.

4.4 Médico residente, profesional de la medicina que ingresa a una unidad médica receptora de residentes para realizar una residencia médica a tiempo completo.

4.5 Profesor adjunto, médico especialista adscrito en la unidad médica receptora de residentes, que coadyuva con el profesor titular en la planeación, conducción y evaluación de la residencia médica correspondiente.

4.6 Profesor titular, médico especialista adscrito en la unidad médica receptora de residentes, con nombramiento de la institución de educación superior, responsable de la planeación, conducción y evaluación de la residencia médica de la institución de salud correspondiente.

4.7 Programa académico, documento emitido por la institución de educación superior que contiene los elementos del plan de estudios de la residencia médica correspondiente.

4.8 Programa operativo, documento que describe las actividades que debe realizar el médico residente, para desarrollar el programa académico de la residencia médica correspondiente en las unidades médicas receptoras de residentes.

4.9 Residencia médica, conjunto de actividades académicas, asistenciales y de investigación que debe cumplir el médico residente dentro de las unidades médicas receptoras de residentes, durante el tiempo estipulado en los programas académico y operativo correspondientes.

4.10 Rotación de campo, conjunto de actividades de carácter temporal contenidas en el programa operativo que debe realizar el médico residente del último año de la residencia médica correspondiente. 
4.11 Sede, principal unidad médica receptora de residentes, que cumple con la infraestructura, equipamiento y plantilla de profesores necesarios para que el médico residente pueda desarrollar la totalidad o la mayor parte de los programas académico y operativo de la residencia médica correspondiente.

4.12 Sistema nacional de residencias médicas, conjunto de dependencias e instituciones de salud responsables de la organización y funcionamiento de las residencias médicas para la formación de médicos especialistas, mediante la coordinación con instituciones de educación superior reconocidas y de conformidad con los objetivos y prioridades del Sistema Nacional de Salud.

4.13 Subsede, unidad médica receptora de residentes, en la que el médico residente puede desarrollar parte de los programas académicos y operativos de la residencia médica correspondiente.

4.14 Unidad administrativa competente, dirección general responsable de conducir la política nacional para la formación, capacitación y actualización de los recursos humanos para la salud; de proponer, promover y vigilar el cumplimiento de las normas oficiales mexicanas en materia de formación, capacitación y actualización de los recursos humanos para la salud; de normar, controlar y evaluar la asignación de campos clínicos y becas de residencias médicas.

4.15 Unidad médica receptora de residentes, establecimiento para la prestación de servicios de atención médica o bien áreas o servicios de la institución de salud que cumplen como escenario académico, en el cual los médicos residentes deben realizar las residencias médicas.

4.16 Unidad médica receptora de residentes para rotación de campo, establecimiento para la prestación de servicios de atención médica donde el médico residente debe realizar su rotación de campo durante el último año de su residencia médica, de acuerdo a lo establecido por la unidad administrativa competente y estipulado en el programa operativo correspondiente.

En la Norma Oficial hay disposiciones referentes a los profesores titular y adjunto que son necesarios en la gestión y los procesos médico-administrativos. Aparecen en el inciso 9 y son las siguientes:
9.1 Cumplir los requisitos académicos y profesionales establecidos por la institución de educación superior que reconoce las residencias médicas

9.2 Cumplir los requerimientos siguientes para ser propuestos por las instituciones de salud como profesor titular o adjunto de una residencia médica en unidades médicas receptoras de residentes:

9.2.1 Ser médico especialista en la disciplina respectiva y contar con cédula con efecto de patente para ejercer profesionalmente la especialidad correspondiente.

9.2.2. Tener experiencia docente de por lo menos un año en la residencia médica correspondiente.

9.2.3 Estar adscrito en la unidad médica receptora de residentes y, en su caso, desempeñar actividades asistenciales inherentes a la residencia médica de que se trate.

9.3 Cumplir durante su estancia en la unidad médica receptora de residentes las actividades siguientes:

9.3.1 Coordinar y supervisar las actividades asistenciales, académicas y de investigación de los médicos residentes, señaladas en el programa operativo correspondiente.

9.3.2 Colaborar en la elaboración del programa operativo de la residencia médica correspondiente, siguiendo los lineamientos de la institución de salud y de la institución de educación superior en su caso; considerando las necesidades en salud de la población relativas a la especialidad médica correspondiente y reforzando la adquisición de destrezas útiles para la aplicación del conocimiento adquirido.

9.3.3 Evaluar el aprendizaje de los médicos residentes, de acuerdo con los programas académico y operativo correspondientes.

9.3.4 Supervisar el desarrollo de los programas académico y operativo de la residencia médica correspondiente.

9.3.5 Evaluar el desempeño de los profesores que participan con él en la residencia médica.

9.3.6 Notificar por escrito a los médicos residentes su situación promovidos o no promovidos, en coordinación con la institución de educación superior correspondiente, de acuerdo con las normas y los procedimientos establecidos por la jefatura de 
enseñanza de las unidades médicas receptoras de residentes.

9.4 El director, subdirector, jefe de enseñanza o jefes de servicio y sus equivalentes de la unidad médica receptora de residentes no deben fungir como profesores titulares.

9.5 Para el desarrollo óptimo de la residencia médica, el profesor titular debe contar con la colaboración de profesores adjuntos, auxiliares, invitados externos, ayudantes $u$ otros de acuerdo a la nomenclatura existente en la unidad médica receptora de residentes.

En el apéndice informativo de la citada Norma se incluye el apartado 10 titulado Actividades clínicas y académicas diarias por servicio, en el que se expresa: «Es el conjunto de acciones que debe realizar el médico residente en el servicio en el que está asignado, incluidas las relacionadas con la docencia, en las que debe de participar activamente». Las acciones se refieren a visita a salas, recepción y entrega de servicios, revisión y elaboración de ingresos, elaboración de historias clínicas, consulta externa, elaboración de notas clínicas, interconsultas, toma de alimentos, guardias, interpretación de estudios, procedimientos diagnósticos y terapéuticos, discusión de diagnósticos, revisión de casos clínicos, conferencias, sesiones bibliográficas, sesiones de departamentales, sesiones generales, talleres, seminarios y días de la semana7. "Estas actividades deberán asignarse con horario y por separado para cada grado académico." El cumplimiento de dichas normativas implica gestión y procesos. En otros términos, la Norma Oficial Mexicana en referencia considera los elementos indispensables para las residencias médicas, expresados en deberes de las instituciones de salud integrantes del Sistema Nacional de Residencias Médicas, apuntando que la Norma tiene como fin garantizar la formación óptima de médicos especialistas, sin menoscabo de la seguridad de los pacientes ni de la atención a la salud de la población.

\section{El médico residente y las competencias educacionales}

Todas las disposiciones de actividades clínicas y docentes que se presentan en el PUEM y en la Norma «para la organización y funcionamiento de residencias médicas» conllevan actividades de gestión y procesos médico-administrativos que se requieren para la organización y el funcionamiento de las residencias médicas en los hospitales. Como complemento a lo mencionado, el International Institute for Medical Education ha señalado siete competencias educacionales necesarias en la atención médica; todas implican gestión y procesos médico-administrativos, y son las siguientes:

1.-Habilidades clínicas.- Los médicos residentes deben diagnosticar y otorgar tratamientos a los pacientes en forma eficiente y efectiva.

2.-Fundamentos científicos de la medicina.- Los médicos residentes deben contar con conocimientos científicos sólidos, con capacidad para aplicar dichos conocimientos a la solución de problemas médicos concretos, debiendo entender los principios en que se basan las decisiones y la atención médica, debiendo adaptarse a los diversos cambios y progresos.

3.-Valores profesionales, actitudes, comportamiento y ética.- Los médicos residentes deben actuar con profesionalismo y conducta ética, debiendo tener presentes los principios de autonomía, beneficencia, no maleficencia y justicia.

4.-Salud pública y sistemas de salud.- Los médicos residentes deben poseer conocimientos en cuanto a promoción de la salud, prevención de enfermedades, así como a su práctica; además, deben conocer la estructura y heterogeneidad de los servicios de salud, y su gestión básica.

5.-Habilidades para analizar, valorar y criticar los conocimientos existentes, la tecnología y la información, empleando en su caso el método científico en la investigación.

6.-Manejo de la tecnología de la información.- Los médicos residentes deben comprender las posibilidades y limitaciones de la tecnología de la información en el estudio de pacientes.

7.-Comunicación.- Los médicos residentes deben tener habilidades de comunicación, entender y comprender a los pacientes y sus familiares, médicos, enfermeras y público en general ${ }^{\circledR}$.

\section{Conclusión}

Los médicos residentes son ejes vertebrales de los servicios clínicos hospitalarios, razón que los obliga a tener conocimientos en la especialidad de su preferencia y además poseer capacidades en gestión, particularmente en microgestión y en los procesos.

La gestión tiene impacto en las actividades de los médicos residentes, fundamentalmente en tres aspectos: el primero es la toma de decisiones, ya que los médicos residentes participan en la resolución de problemas y al tener conocimientos de gestión tomarán mejores decisiones médico-administrativas; el segundo 
aspecto es la gestión de los recursos, que se refiere a la capacidad y el talento requeridos para la dirección adecuada de un equipo de trabajo integrado por el capital humano, lo más valioso de la organización, el uso racional de los recursos materiales, físicos, financieros y tecnológicos; y el último aspecto hace referencia a los procesos médico-administrativos, que deben obedecer a un orden y lógica, facilitando de esta forma la impartición de los servicios. Es decir, si se hace partícipe en la gestión clínica al médico residente, se contribuye a mejorar los servicios médicos, que serán otorgados con mayor eficacia y eficiencia. Cabe señalar que el objetivo de la gestión clínica por parte de los residentes es ofrecer un servicio de calidad y seguridad para el paciente y su familia, siempre con ética y humanismo.

\section{Financiamiento}

Los autores declaran que no fue necesario financiamiento.

\section{Conflicto de intereses}

Los autores declaran que no hubo conflicto de intereses.

\section{Bibliografía}

1. Dal Bó A. Hospitales de reforma. Buenos Aires, Argentina: Editorial Biblos; 2008. p. 46.

2. Secretaría del Trabajo y Previsión Social. Ley Federal del Trabajo. México; 2012. p. 28

3. Lozano Kasten F. de J. Gestión hospitalaria. Macrogestión, mesogestión y microgestión. En: Martínez Ramírez A, coordinador. Gestión hospitalaria. Guadalajara, Jalisco, México: IDEA; 2007. p. 31.

4. Zanovello AL. Qualidade aplicada aos seviços de saude. En: Hoteleria hospital. Boeger MM. Coordinador. Barueri, São Paulo: Manole; 2011. p.75

5. Álvarez-Hernández G, Medécigo-Vite S, Ibarra-García C. Prevalencia del síndrome de desgaste profesional en médicos residentes de un hospital pediátrico en el Estado de Sonora. Boletín Médico del Hospital Infantil de México. 2010;67:15-23.

6. Plan Único de Especializaciones Médicas (PUEM). Facultad de Medicina, Universidad Nacional Autónoma de México. México;

7. Secretaría de Salud. Norma Oficial Mexicana NOM-001-SSA3-2012. Educación en salud para la organización y funcionamiento de residencias médicas. México; 2012. p. 5-12.

8. Institute for International Medical Education, Core Committee. Global minimum essential requirements in medical education. Medical Teacher 2002;24:130-5. Traducción al español en Educación Médica. 2003;6(Supl 2):11-9 